\title{
Illegal Immigration and the Shadow Economy
}

C. Camacho, F. Mariani and L. Pensieroso

Discussion Paper 2015-14

\section{Institut de Recherches Économiques et Sociales de l'Université catholique de Louvain}




\title{
Illegal Immigration and the Shadow Economy*
}

\author{
Carmen Camacho $^{\dagger} \quad$ Fabio Mariani $^{\ddagger} \quad$ Luca Pensieroso ${ }^{\S}$
}

June 29, 2015

\begin{abstract}
We build a general equilibrium model in which both illegal immigration and the size of the informal sector are endogenously determined and interact in a non-trivial way. We show that policy measures such as tax reduction and detection of informal activities can be used as substitutes for border enforcement, in order to contrast illegal immigration. In our framework, a welfare-maximising Government will never choose to drive illegal immigration to zero, but will set the tax rate to a lower value if it includes illegal immigration in its objective function.
\end{abstract}

JEL Classification: O17; F22; J61.

Keywords: Illegal immigration; Clandestine workers; Informal sector; Shadow economy; Black market; Taxation; Immigration policy

*Paper presented at the Shadow 2011 conference in Muenster and at the PET 2012 conference in Taipei. We thank the participants at these meetings as well as the participants in a seminar at the University of Louvain (UCLouvain) for interesting remarks. Frédéric Docquier and Pierre Pestieau made useful remarks on an earlier version. The usual disclaimer applies.

${ }^{\dagger}$ CNRS and Paris 1, Panthéon-Sorbonne. E-mail: maria.camacho-perez@univ-paris1.fr

łIRES, Université catholique de Louvain; IZA, Bonn. E-mail: fabio.mariani@uclouvain.be

§IRES, Université catholique de Louvain. E-mail: luca.pensieroso@uclouvain.be 


\section{Introduction}

What is largely fueling the underground economy, experts say, is the nation's growing ranks of low-wage, illegal immigrants.

(The Wall Street Journal, Classroom edition, April 2005 http://www . wsjclassroomedition.com/archive/05apr/econ_underground.htm)

For many undocumented immigrants, the underground economy is the only means of finding a job.

(OECD Observer 219, 1999 http://www.oecdobserver.org/news/ fullstory.php/aid=190)

Illegal immigration is a controversial issue in political debates across Europe and beyond. Much of the debate focuses on the border-enforcement measures to control the inflow of illegal migrants. In this paper, we shall take a different perspective and focus instead on the incentives that make illegal immigration both attractive to potential immigrants and convenient for native firms. In particular, we deal with the role played by the shadow economy in the destination country.

Although it is common belief that illegal immigration and the shadow economy are somehow linked to each other, the economic literature is surprisingly silent on the theme. There exists a large and growing literature on the shadow economy (Amaral and Quintin (2006), Dabla-Norris et al. (2008), Ihrig and Moe (2004), Schneider and Enste (2000), Tanzi (1983) and Tanzi (1999) among others), which focuses on the main determinants of informality, but by and large neglects the role of illegal immigration. Several other studies deal with illegal migration (see Borjas (1994), Djajic (1997), Djajic and Vinogradova (2013), Djajic and Vinogradova (2015), Hazari and Sgro (2003) among others), but again with no specific focus on its interplay with the underground economy. Our paper bridges this gap, building a general equilibrium model of the destination country, in which both illegal immigration and the size of the informal sector are endogenously determined. 1

In our model, prospective immigrants react to wage differentials between the source and the destination country, taking also into account how their employment opportunities depend on their legal status. In particular, as a consequence of their status, potential illegal immigrants face a positive probability of deportation when crossing the border and can only work in the informal sector in the destination country.

\footnotetext{
${ }^{1}$ The closest approach to our work is that by Dell'Aringa and Neri (1987), who study the effects of an exogenous increase of immigration on the labour market in Italy.
} 
Firms in the destination country choose between operating in the formal or in the informal sector. This choice depends on the availability of illegal immigrant workforce, but is also driven by the usual determinants of informality - such as taxation, differential productivity, detection costs. In particular, we introduce public expenditure as an input in the production function as in Barro (1990), and assume that firms in the informal sector have only partial access to it. The presence of productive public expenditures introduce an important trade off in the model. On the one hand, higher public expenditure needs to be financed through higher taxes, which pushes firms to operate informally. On the other hand, if too many firms enter the shadow economy, there will be an erosion of the tax base. This implies less public expenditures, and therefore less production in the whole economy.

In this framework, illegal immigration and the size of the informal sector interact in a non-trivial way. In particular, they may be strategic complements: the presence of a widespread informal sector can foster illegal immigration, while the presence of many illegal aliens can strengthen the incentive for firms to go informal.

A major implication of our analysis is that tax reductions and fiscal controls can both be used as alternatives to border enforcement, if the Government wants to reduce illegal immigration. Due to the general equilibrium structure of the model, we can also say something about the relative effectiveness of the above policy measures. For instance, if informality and illegal immigration are strategic complements, fiscal controls turn out to be more effective than border enforcement at reducing illegal immigration. ${ }^{2}$

Our model also allows for a more general policy analysis, where contrasting illegal immigration is not an objective per se, but is instrumental to maximising social welfare. In particular, we study the welfare-maximising tax rate under three different specifications of a fairly general social welfare function: a benchmark function, in which only the utility of legal workers matters; a 'xenophobic' function, in which the relative size of illegal immigration affects social welfare negatively; and an 'altruistic' function, where the utility of illegal aliens is also taken into account. Results show that the Government will set a higher tax rate in the benchmark case, i.e. when it focuses exclusively on the welfare of legal workers, than in all the other cases. In general, our analysis shows that the welfare-maximising level of informality is different from zero, thus implying a positive quantity of

\footnotetext{
${ }^{2}$ In the appendix, we work out a numerical exercise based on an extended version of the model, in which border enforcement and fiscal controls are both financed out of the Government budget constraint. In this case, we show that tighter border controls might even be associated with larger flows of illegal immigration.
} 
illegal immigrants.

The remainder of the paper is organized as follows. In Section 2, we present and discuss our model. In Section 3, we develop the policy implications of our analysis. Section 4 concludes.

\section{The model}

The economy produces only one good. There are two single-firm sectors in the economy, a formal sector and an informal one, indexed by $F$ and $I$, respectively. The Government levies taxes on formal production to provide a public good $G$ that enters the production function of the two sectors. We assume that the informal sector does not pay taxes but, because of its status, cannot fully benefit from public services. Total production is $Y=Y_{F}+Y_{I}$.

\subsection{Production}

Production in the formal sector takes place according to the following Cobb-Douglas function:

$$
Y_{F}=A_{F} G^{\alpha} L_{F}^{1-\alpha},
$$

where $A$ stands for total factor productivity, $G$ denotes public expenditure and $L$ is the labour input. The after-tax value of production in the formal sector is:

$$
J_{F}=(1-\tau) Y_{F},
$$

where $\tau \in[0,1]$ is the tax rate. Taxes are used to finance the production of the public good, as described by the budget constraint of the Government:

$$
G=\tau Y_{F} .
$$

The informal sector produces according to:

$$
Y_{I}=A_{I}(\delta G)^{\alpha} L_{I}^{1-\alpha},
$$

where $\delta \in(0,1)$ captures the idea that the firm operating informally cannot have full access to public services (e.g. infrastructures, public subsidies, etc.). For analytical convenience, we assume that the two sectors have the same factor shares.

The firm in the shadow economy does not pay taxes. It can, however, incur in detection by fiscal authorities, which occurs with exogenous probability $\lambda \in(0,1)$. Detection implies destruction of the whole production. Accordingly, the expected value of production in the informal sector is:

$$
J_{I}=(1-\lambda) Y_{I} .
$$


Notice that $\lambda$ can also be interpreted as the cost of avoiding detection, measured as a fraction of output ${ }^{3}$

The labour force is made of native and immigrant workers. The latter can enter the country either legally or illegally. Legal migration results from a quota decided by the Government. We assume that the quota is always fulfilled. Accordingly, the number of legal migrants $M$ is

$$
M=q N,
$$

where $N$ stands for the native population, and $q \in(0,1)$ is the quota. Hence, the total legal workforce $P$ is given by

$$
P=(1+q) N \text {. }
$$

Unlike legal immigrants, illegal immigrants can only be employed by the informal firm. The number of illegal immigrants is denoted by $Z$, and will be endogenously determined by the model.

We assume that legal immigrants and natives - the two components of the legal labour force - are perfect substitutes in production functions (1) and (4), while illegal immigrants and the legal labour force are imperfect substitutes. ${ }^{4}$ Accordingly, calling $\rho$ the share of the legal labor force working in the informal sector, the labour inputs in the two sectors can be written as

$$
L_{F}=(1-\rho) P
$$

and

$$
L_{I}=(\rho P)^{\beta} Z^{1-\beta},
$$

respectively.

\subsection{Profit maximization}

The formal firm chooses labour so as to maximize its after-tax profits:

$$
\max _{L_{F}} J_{F}-w_{F} L_{F}
$$

\footnotetext{
${ }^{3}$ In the benchmark version of the model, $\lambda$ is exogenous. We will remove this assumption in the appendix.

${ }^{4}$ Our formulation conforms to Borjas et al. (2011), who argue in favour of perfect substitutability between legal immigrants and natives. A recent article by Peri (2011) finds that the elasticity of substitution between equally-skilled legal immigrants and natives is very high. For what concerns the degree of substitutability between illegal immigrants and natives in the informal sector, to the best of our knowledge there is no empirical evidence upon which we can rely. However, as suggested by Hazari and Sgro (2003), we feel it is realistic to have less-than-perfect substitutability between legal and illegal workforce. The Cobb-Douglas formulation in 99 is a convenient way to introduce this hypothesis in the model.
} 
The first-order condition of problem (10) reads

$$
w_{F}=\frac{\partial J_{F}}{\partial L_{F}}=(1-\tau)(1-\alpha) A_{F} G^{\alpha} L_{F}^{-\alpha} .
$$

Substituting Equation (3) for $G$, we obtain

$$
w_{F}=(1-\tau)(1-\alpha)\left(A_{F} \tau^{\alpha}\right)^{\frac{1}{1-\alpha}},
$$

which does not depend on $L_{F}$. This is due to the fact that, once the Government budget constraint is taken into account, the formal sector's technology becomes linear in labour. 5

The firm operating in the informal sector free-rides on the public good. This implies that $J_{I}$ is not linear in labour anymore. Wages will include not only the marginal productivity of labour but also the marginal productivity of public expenditures, which in turn depends on labour in the formal sector (see Equation (3)). We assume that a share $\mu$ of the marginal productivity of public expenditures is grasped by legal workers, while $(1-\mu)$ goes to illegal immigrants. Therefore, the wage of legal workers in the informal sector is

$$
w_{I}=\frac{\partial J_{I}}{\partial(\rho P)}+\mu \frac{\partial J_{I}}{\partial G} \frac{G}{\rho P}
$$

where $\mu \in(1 / 2,1)$ can be interpreted as a measure of the exploitation of illegal immigrants. By the same token, the wage of illegal workers in the informal sector is

$$
w_{Z}=\frac{\partial J_{I}}{\partial Z}+(1-\mu) \frac{\partial J_{I}}{\partial G} \frac{G}{Z} \text {. }
$$

\subsection{Illegal migration}

We assume that in the source country workers are paid an exogenous constant wage $\omega<w_{F}$. This implies that the quota of legal migrants chosen by the destination country is always fulfilled. Those workers who fall outside the quota can still migrate illegally. To do so, they incur in a fixed migration $\operatorname{cost} c$, and, if caught at the border and deported, they face a penalty $x]^{6}$ The probability of being caught at the border is called $\eta$.

\footnotetext{
${ }^{5}$ In fact, formal labour contributes to formal production both directly and indirectly through $G$. Notice that in the private economy formal workers do not fully internalize the social effects of public expenditures.

${ }^{6}$ The deportation penalty $x$ is meant to capture the social stigma or effective punishment (like jailing, property seizure and the like) in which deported migrants can incur. Besides, given our simple linear formulation in Equation (15), the introduction of $x$ preserves a role for the variable $\eta$, that would otherwise disappear.

${ }^{7}$ In the benchmark version of the model, $\eta$ is exogenous. We will remove this assumption in the appendix.
} 
Accordingly, a prospective illegal migrant will try to access the destination country as long as

$$
(1-\eta) w_{Z}+\eta(\omega-x)-c \geq \omega
$$

that is, if the expected return from illegal migration exceeds the domestic wage. We assume that the pool of potential migrants is large enough for Equation (15) to hold as an equality.

\subsection{Equilibrium}

In the destination country, legal workers are perfectly mobile across sectors. The equilibrium condition on wages, $w_{F}=w_{I}$, thus determines $\rho$ as a function of $Z$, that is how the legal labour force is split between the two sectors depending on the number of illegal immigrants. Since we cannot get an analytical solution for $\rho(Z)$, we solve $w_{F}=w_{I}$ for $Z$ and call the solution $Z_{1}$ :

$$
Z_{1}(\rho)=N(1+q)\left(\frac{(1-\alpha)(1-\tau) A_{F} \delta^{-\alpha} \rho^{1-(1-\alpha) \beta}(1-\rho)^{-\alpha}}{A_{I}(1-\lambda)[(1-\alpha) \beta+\alpha \mu]}\right)^{\frac{1}{(1-\alpha)(1-\beta)}} .
$$

On the other hand, since $w_{Z}$ depends upon the number of legal workers employed in the informal sector, condition (15) implies that the number of potential illegal immigrants is in turn influenced by the value of $\rho$. Indeed, solving Equation (15) for $Z$, and noticing that only a fraction $(1-\eta)$ of the potential illegal immigrants will actually reach destination, we get a second expression of $Z$ as a function of $\rho$ :

$$
\begin{aligned}
& Z_{2}(\rho)= \\
& N(1+q)\left(\frac{(1-\lambda)[1-((1-\alpha) \beta+\alpha \mu)] A_{I}\left(\tau A_{F}\right)^{\frac{\alpha}{1-\alpha}} \delta^{\alpha} \rho^{(1-\alpha) \beta}(1-\rho)^{\alpha}}{\omega+\frac{c+\eta x}{1-\eta}}\right)^{\frac{1}{\alpha+(1-\alpha) \beta}} .
\end{aligned}
$$

It can be checked that while $Z_{1}(\rho)$ is strictly increasing and convex, $Z_{2}(\rho)$ is concave, increasing from 0 to $\bar{\rho}$ and decreasing thereafter, where

$$
\bar{\rho}=\frac{(1-\alpha) \beta}{\alpha+(1-\alpha) \beta} .
$$

Equations (16) and (17) form a two-dimension system in $Z$ and $\rho$, whose solution is the equilibrium of the model. Proposition 1 proves the existence and uniqueness of the solution. 
Proposition 1 The system formed by Equations (16) and (17) has a unique solution $\left(\rho^{*}, Z^{*}\right)$ :

$$
\begin{aligned}
\rho^{*} & =\frac{1}{1+\kappa^{\prime}} \\
Z^{*} & =\frac{N(1+q)}{(1+\kappa)}\left(\frac{A_{F}(1-\alpha)(1-\tau) \delta^{-\alpha} \kappa^{-\alpha}}{A_{I}(1-\lambda)[(1-\alpha) \beta+\alpha \mu]}\right)^{\frac{1}{(1-\alpha)(1-\beta)}},
\end{aligned}
$$

with

$$
\mathcal{\kappa}^{-\alpha}=\frac{A_{F}^{-\beta} A_{I}(1-\lambda) \delta^{\alpha} \tau^{\alpha(1-\beta)}}{(1-\tau)^{(\alpha(1-\beta)+\beta)}}\left(\beta+\frac{\alpha \mu}{1-\alpha}\right)^{\alpha(1-\beta)+\beta}\left(\frac{1-(1-\alpha) \beta-\alpha \mu}{\omega+\frac{c+\eta x}{1-\eta}}\right)^{(1-\alpha)(1-\beta)} .
$$

Proof

See Appendix.

We can then claim the following.

Lemma 1 Illegal immigration and the informal sector are strategic complements, if $\rho^{*}<\bar{\rho}$.

Proof

The result follows from Proposition 1 and the shape of the functions $Z_{1}(\rho)$ and $Z_{2}(\rho)$.

Hence, if $\rho^{*}<\bar{\rho}$, the bigger the informal sector, the higher the incentives for potential migrants to enter the country illegally. Furthermore, the higher the number of illegal immigrants, the larger the relative return to informal production.

Whether strategic complementarity arises or not depends on the parameters of the model. In Equation (18), the value $(1-\alpha) \beta$ is the legal labour share in the informal sector: the higher this share, the higher $\bar{\rho}$ and the more likely the case of strategic complementarity. Therefore, countries in which native workers and legal immigrants contribute substantially to the informal sector are more likely to be characterised by strategic complementarity. In addition, Proposition 1 implies that there exist threshold values $\bar{\eta}, \bar{\lambda}, \bar{\delta}$ such that $\rho^{*}<\bar{\rho}$, if either $\eta<\bar{\eta}$, or $\lambda<\bar{\lambda}$, or $\delta<\bar{\delta}$.

The two alternative cases of strategic complementarity and substitutability are depicted in Figure 1 .

\subsection{Comparative statics}

We now turn to comparative statics, in order to understand how the parameters of the model affect the equilibrium values of illegal immigration and the size of the shadow economy. 


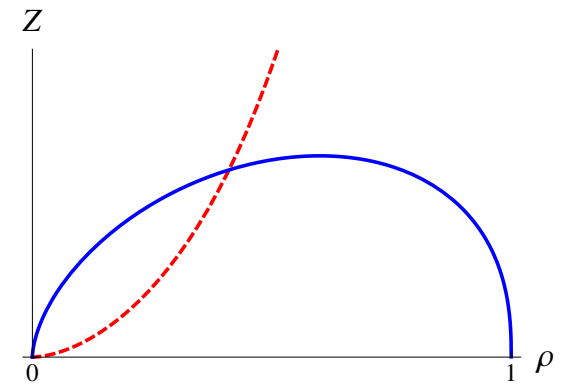

(a) $\rho^{*}<\bar{\rho}$ : complementarity

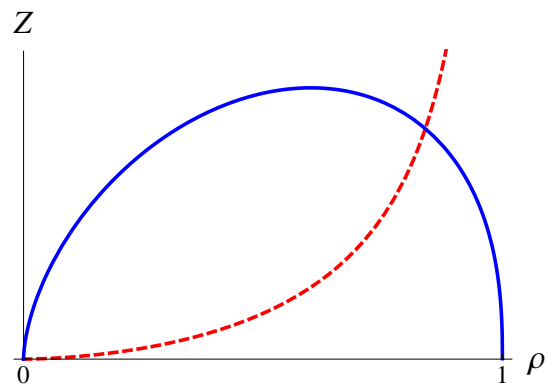

(b) $\rho^{*}>\bar{\rho}$ : substitutability

Figure 1: Equilibrium. $Z_{1}(\rho)$ (red-dashed line), $Z_{2}(\rho)$ (blue-solid line).

Proposition 2 Given $\rho^{*}$ and $Z^{*}$ as specified in Proposition 1 .

(i) $\frac{\partial Z^{*}}{\partial \lambda}<0$ and $\frac{\partial \rho^{*}}{\partial \lambda}<0$;

(ii) $\frac{\partial Z^{*}}{\partial \eta}<0$ and $\frac{\partial \rho^{*}}{\partial \eta}<0$;

(iii) $\frac{\partial Z^{*}}{\partial x}<0$ and $\frac{\partial \rho^{*}}{\partial x}<0 ; \frac{\partial Z^{*}}{\partial c}<0$ and $\frac{\partial \rho^{*}}{\partial c}<0$;

(iv) $\frac{\partial Z^{*}}{\partial q}>0$ and $\frac{\partial \rho^{*}}{\partial q}=0$;

(v) $\frac{\partial \rho^{*}}{\partial \tau}>0$ and $\exists \hat{\tau}=\alpha \frac{(1+\beta \kappa) \alpha+(1-\beta) \kappa}{(1+\beta \kappa) \alpha+(\alpha-\beta) \kappa}>0$, such that
(v.1) $\frac{\partial Z^{*}}{\partial \tau}(\hat{\tau})=0$
(v.2) if $\tau<\hat{\tau}, \frac{\partial Z^{*}}{\partial \tau}>0$;
(v.3) if $\tau>\hat{\tau}, \frac{\partial Z^{*}}{\partial \tau}<0$.

Proof

Computing the partial derivatives of Equations (19) and (20) leads to the results.

According to Proposition 2, the equilibrium number of illegal immigrants (i) decreases with the detection probability of informal activity, (ii) 
decreases with the probability of deportation for illegal migrants, (iii) decreases with the penalty associated to unsuccessful migration and with the fixed cost of migration, (iv) increases with the migration quota. In cases (i) to (iii), changes in the parameters have the same effect on $\rho^{*}$ as they have on $Z^{*}$. In case (iv), instead, the size of the informal sector is unaffected by variations in the migration quota, since the latter has no bearing on the relative return to informality.

As far as illegal immigration is concerned, the rationale behind results (ii) and (iii) of Proposition 2 is straightforward, as all the parameters involved directly affect the decision to migrate. For what concerns point (i), instead, the negative impact of $\lambda$ on illegal immigration stems from a general equilibrium effect: more severe fiscal controls reduce the incentive for firms to operate informally, thereby reducing the demand for illegal immigrants. As far as (iv) is concerned, the positive effect of $q$ on $\mathrm{Z}$ depends on the complementarity between legal and illegal workers in the informal sector, and the assumption that the number of prospective migrants is infinite. Finally, the effect of $\tau$ on $Z^{*}$ described in $(v)$ is non-monotonic. On the one hand, an increase in $\tau$ raises the relative return to informal production, thereby increasing $Z^{*}$. On the other hand, changes in $\tau$ also entail general equilibrium effects through variations of $G$ (see Equation (3)). For low levels of taxation, increases in the tax rate augment the resources available to the Government for public expenditure. Again, this calls for higher $Z^{*}$ because higher public expenditure increases the marginal productivity of illegal immigrants. However, when the tax rate becomes too high, that is for $\tau>\hat{\tau}$, further increases in taxation lower production in the formal sector, thereby eroding the tax base, and hence making $G$ decrease. This in turn decreases the marginal productivity of labour by illegal immigrants, reducing their incentives to migrate ${ }^{8}$

\subsection{The size of the shadow economy}

The variable $\rho$ measures the relative size of the shadow economy in terms of labour. If instead we want to measure the underground economy as a fraction of GDP, the appropriate measure of the relative size of the shadow economy in our model would be

$$
\sigma \equiv \frac{Y_{I}}{Y_{F}+Y_{I}}
$$

By replacing $Y_{I}$ and $Y_{F}$ with their values for $Z=Z^{*}$ and $\rho=\rho^{*}$, the

\footnotetext{
${ }^{8}$ Thus, we obtain a sort of migration Laffer curve.
} 
equilibrium value of $\sigma$ can be simplified into

$$
\sigma^{*}=\frac{1}{1+\frac{1-\rho^{*}}{\rho^{*}} \frac{(1-\lambda)[(1-\alpha) \beta+\alpha \mu]}{(1-\alpha)(1-\tau)}} .
$$

Expression (23) implies that $\partial \sigma^{*} / \partial \rho^{*}>0$, so that the comparative statics of $\sigma^{*}$ and $\rho^{*}$ are qualitatively the same.

\section{Policy}

The general equilibrium structure of the model discussed here above allows us to draw some interesting policy implications. We will first see how the Government can use alternative instruments in order to regulate the inflow of (illegal) foreign migrants, and then turn to a more general welfare analysis.

\subsection{Tackling illegal immigration}

If the Government just wants to reduce the flow of illegal immigrants, Proposition 2 implies that it is not constrained to rely on border controls, but it can also resort to alternative policies, namely lowering taxes and increasing fiscal controls. In other words, our model suggests that the reach of fiscal policy goes beyond its traditional domain, and fiscal instruments can be effectively used as immigration policy tools.

Given this, one may also want to compare the relative effectiveness of alternative policy instruments. In our setting, border controls affect the supply of illegal immigration, as $Z_{2}(\rho)$ is a function of $\eta$. However, they do not affect the demand, since $Z_{1}(\rho)$ does not depend on $\eta$. For what concerns fiscal controls, $\lambda$ affects both demand and supply of illegal immigration, as it enters in both the expressions for $Z_{1}$ and $Z_{2}$ in Equations (16) and (17). This suggests that fiscal controls may be more effective than border controls at reducing illegal immigration, as stated in the following Proposition.

Proposition 3 Assume that illegal immigration and the informal sector are strategic complements (Lemma 1). Then,

(i) if $\lambda \geq \eta,\left|\frac{\partial Z^{*}}{\partial \lambda}\right|>\left|\frac{\partial Z^{*}}{\partial \eta}\right|$; 
(ii) if $\lambda<\eta$, there exists $\bar{c}=\frac{(1-\eta)^{2} \omega-[(1-\lambda)-\eta(1-\eta)] x}{\eta-\lambda}$ such that for $c<\bar{c},\left|\frac{\partial Z^{*}}{\partial \lambda}\right|>\left|\frac{\partial Z^{*}}{\partial \eta}\right|$.

Proof: See the Appendix.

Proposition 3 reveals that, under strategic complementarity, increasing $\lambda$ may be more effective than increasing $\eta$ at reducing illegal immigration, provided that the probability of detecting informal activity in the domestic economy is higher than the probability of detecting illegal immigrants at the border, i.e. if $\lambda \geq \eta$. When $\lambda<\eta$, fiscal controls are still more effective than border controls, if the cost of migrating illegally is sufficiently low.

The differential effectiveness of the two instruments hinges on two characteristics of the model. First, as mentioned above, while $\lambda$ affects both the incentive for firms to go informal and the incentive to migrate illegally, $\eta$ influences only the latter. Second, restricting our attention to the incentive to migrate, we can see that the effect of $\lambda$ is negative, while $\eta$ has an ambiguous effect. To understand why, consider the incentive to migrate illegally, as expressed by Equation (15). The higher is $\lambda$, the smaller the marginal productivity of labour in the informal sector. This implies a lower $w_{Z}$, and thereby a lower expected reward from illegal immigration. The effect of $\eta$ is twofold. First, a higher $\eta$ reduces the expected reward from illegal immigration, by reducing the probability of earning the wage $w_{Z}$. Second, as fewer immigrants will actually reach the destination country, their marginal productivity in the informal sector will be higher, which goes against the first effect, implying a higher $w_{Z}$.

As far as the tax rate $\tau$ is concerned, its relative effectiveness with respect to border and fiscal controls is difficult to assess. First, different from $\eta$ and $\lambda$, the effect of $\tau$ on the equilibrium value of illegal immigration $Z^{*}$ in non-monotonic, as stated in Proposition 2 . Second, since $\eta$ and $\lambda$ are probabilities, while $\tau$ is a tax rate, these two types of variables are inherently heterogeneous, and therefore not directly comparable.

These results abstract from the possible trade off between alternative policies. In particular, fiscal and border controls are typically financed out of the same scarce resources, namely fiscal receipts. Moreover, public funds can be transformed into the probabilities $\lambda$ and $\eta$ according to different technologies, that are not specified in the current version of the model. 9$]$

\footnotetext{
${ }^{9}$ In the Appendix, we propose an extended version of the model who gets closer to reality, by assuming that border control and detection of informal activity are police-
} 


\subsection{Welfare}

Our discussion so far has stemmed from the hypothesis that the Government would like to decrease the number of illegal aliens in the domestic economy. Although this is arguably what can be inferred from current political debates, it is hardly a logical implication of the model. Rather than being an objective in itself, the regulation of illegal immigration should in fact be instrumental to maximising social welfare. As we shall see below, if public expenditure is productive, the welfare-maximising level of informality will be different from zero, thus implying a positive quantity of illegal immigrants. In other words, zero illegal immigration is not likely to be the chosen outcome of a welfare-maximising Government. ${ }^{10}$

To study the issue, we assume that the Government maximises the following welfare function:

$$
\Omega=\frac{P}{P+\psi Z^{*}}\left(w_{F}^{*}-\theta \frac{Z^{*}}{P}\right)+\frac{\psi Z^{*}}{P+\psi Z^{*}} w_{Z}^{*} .
$$

In this formulation, the Government values a weighted average of percapita consumption by native, legal and illegal immigrants. ${ }^{11}$ We assume that the Government gives the same weight to native and legal immigrants (i.e. 1), whereas it gives a weight $\psi \in[0,1]$ to illegal immigrants. This parameter can be interpreted as the degree of altruism of voters towards disenfranchised illegal immigrants. Moreover, we assume that the relative size of illegal immigration might exert a direct negative effect on the utility

operated, and therefore financed through fiscal recipes. Such a feature introduces a trade off between border patrolling and detection of informal activity, in the context of a balanced Government budget. This model could only be solved numerically. Results from simulations confirm our analytical findings, and even reinforce them. Indeed, quite paradoxically, a Government enforcing more border patrolling with the intent of reducing the number of illegal aliens in the country might instead end up having more of them. This stems from the fact that in the model any increase in border patrolling has the side effect of reducing detection of informal activities, which in turn increases the returns to the informal sector, thereby acting as a call for potential immigrants to migrate illegally.

${ }^{10}$ Our Government differs from a benevolent dictator, concerned with the implementation of the social optimum. Unlike a full-fledged social planner, the Government here takes the structure of incentives that determine the market equilibrium as given, without internalizing the externality due to the presence of the public expenditure G. Accordingly, we are in a second-best scenario. The underlying assumption that we have made for analytical tractability is that the Government chooses its policy variables by aggregating individual preferences. This does not impinge on the overall validity of our the analysis, since the main focus of this article is on illegal immigration and not on optimal taxation perse.

${ }^{11}$ Given that in our model there is no capital, maximizing per capita consumption is at equilibrium tantamount to maximizing wages. 
of native voters (xenophobic preferences), through the parameter $\theta \geq 0$, which accounts for direct aversion towards illegal immigration.

The description of social welfare in Equation (24) is fairly general, and yet too complex to allow for the immediate derivation of clear-cut analytical results. Therefore, we have chosen to decline this social welfare function into different sub-cases, depending on the values of the parameters $\theta$ and $\psi$ that describe the different possible attitudes of the society towards (illegal) immigration. This will allow us to derive interesting analytical results for public policy. We shall then come back to the original welfare function and consider what we have learned.

As a benchmark case we consider a welfare function given by Equation (24) for $\theta=0$ and $\psi=0$ :

$$
\Omega_{b}=w_{F}^{*}=A_{F}^{\frac{1}{1-\alpha}}(1-\alpha)(1-\tau) \tau^{\frac{\alpha}{1-\alpha}},
$$

where subscript $b$ stand for 'benchmark'. This formulation is tantamount to assuming that the Government maximises per capita consumption of legal workers (natives plus legal immigrants), without taking into account neither the number nor the consumption of illegal immigrants. The reason for considering this as the benchmark case is that illegal immigrants do not have voting rights, and therefore might not enter the objective function of the Government. This function has three interesting characteristics. First, $\Omega_{b}$ is a hump-shaped function of $\tau$ and reaches a maximum at $\tau_{b}=$ $\alpha$. Accordingly, for $\tau=\tau_{b}$, the welfare maximising number of illegal immigrants is strictly positive. Second, $\Omega_{b}$ does not depend on $\lambda$ or $\eta$. Third, given $\hat{\tau}$ from Proposition 2 , i.e. the level of $\tau$ below which the number of illegal immigrants always varies in the same direction as the tax rate, we can prove that $\tau_{b}$ is always smaller than $\hat{\tau}$.

Lemma 2 Given $\hat{\tau}$ from Proposition 2 if $\hat{\tau}>0$, then $\tau_{b}<\hat{\tau}$.

Proof

See Appendix.

The second declination of our welfare function still ignores consumption by illegal immigrants (i.e. $\psi=0$ ), but includes the possibility that native voters have a negative sentiment against illegal migrants, i.e. $\theta>0$. Accordingly, Equation (24) becomes,

$$
\Omega_{x}=\Omega_{b}-\theta \frac{Z^{*}}{P}
$$

where subscript $x$ stands for 'xenophobic'. It can be noticed that for $\theta=0$, $\Omega_{x}$ coincides with $\Omega_{b}$. 
By comparing $\Omega_{x}$ with $\Omega_{b}$, we have a second policy implication: a welfare maximising Government who has xenophobic preferences will set a lower tax rate than a Government who simply disregards illegal immigrants ${ }^{12}$ The following proposition makes the point.

Proposition 4 Be $\tau_{x}$ the value of $\tau$ which maximizes $\Omega_{x}$, then $\tau_{x} \leq \tau_{b}$.

Proof

See Appendix.

To understand the rationale behind this result, consider the case of strategic complementarity ${ }^{13}$ The social welfare function $\Omega_{x}$ depends negatively on $Z^{*}$, which in turn is a positive function of $\tau$ (since the incentive to go informal increases with taxes). Suppose the tax rate is set at the level that maximizes $w_{F}^{*}$. Then, looking at Equations (25) and (26), one can see that there is room for welfare improvement through a reduction in taxes: a xenophobic policy maker is willing to trade off a decrease in wages against a decrease in the number of illegal immigrants.

The third declination of our social welfare function includes no xenophobic component (i.e. we are back to the case $\theta=0$ ), but on the contrary encompasses the well-being of illegal immigrants. In terms of the parameters of Equation (24), this translates into the assumption $\psi>0$. Accordingly, the social welfare function becomes

$$
\Omega_{a}=\frac{P w_{F}^{*}+\psi Z^{*} w_{Z}^{*}}{P+\psi Z^{*}}
$$

where subscript $a$ stands for 'altruistic'. For $\psi=0, \Omega_{a}$ boils down to $\Omega_{b}$. For $\psi=1$, illegal immigrants are given the same weight as all other workers, a situation we could label as perfect altruism. To ease comparison, we can rewrite Equation (27) as

$$
\Omega_{a}=\Omega_{b}-\frac{\psi Z^{*}}{P+\psi Z^{*}}\left(w_{F}^{*}-w_{Z}^{*}\right)
$$

which allows us to claim the following:

Proposition 5 Be $\tau_{a}$ the value of $\tau$ which maximizes $\Omega_{a}$, then $\tau_{a} \leq \tau_{b}$.

\footnotetext{
${ }^{12}$ Therefore, our model provides a rationale for the common observation that xenophobic parties typically advocate lower taxes.

${ }^{13}$ The result of the proposition is actually more general, as it applies also to the case of substitutability. See the proof in the Appendix.
} 
Proof

See Appendix.

Hence, introducing altruism towards illegal immigrants implies that the welfare-maximizing tax rate is again lower than in the benchmark case. This result is qualitatively the same as Proposition 4. This might be surprising: $\Omega_{x}$ introduces xenophobic preferences, while $\Omega_{a}$ allows for a certain degree of altruism towards illegal immigrants, and yet, in both cases the welfare-maximising tax rate is lower than in the benchmark case. To understand the logic of this seemingly puzzling result, consider again the case of strategic complementarity. Given that $\Omega_{a}$ is positively related to the average wage of illegal immigrants, which is in turn a negative function of $Z^{*}$, the Government prefers fewer, but wealthier illegal immigrants. ${ }^{14}$ Therefore, it will reduce taxes below $\tau_{b}$, so as to decrease the size of the informal sector, and then discourage illegal immigration $\sqrt{15}$ The social welfare functions $\Omega_{b}, \Omega_{x}$ and $\Omega_{a}$ are represented in Figure 2 .

As far as the general welfare function $\Omega$ is concerned, the analysis of the three different sub-cases - benchmark, xenophobic and altruistic - allow us to prove the following.

Proposition 6 There exists $\tau^{*}$ such that $\Omega$ is maximized, and $\tau^{*} \leq \min \left\{\tau_{a}, \tau_{b}, \tau_{x}\right\}$.

Proof

See Appendix.

To grasp the logic behind Proposition 6, consider that - as explained in Propositions 4 and 5 - the introduction of altruism or xenophobia makes the welfare-maximizing tax rate decrease with respect to the benchmark case. A fortiori, when we consider a social welfare function encompassing both altruism and xenophobia, the welfare-maximizing tax rate will be lower than the benchmark case.

\footnotetext{
${ }^{14}$ The results of Proposition 5 do not rest on the assumption of strategic complementarity. They do depend, however, on the way we have introduced altruism towards illegal immigrants in Equation (27). Alternative specifications are possible. In particular, the relevant variable for altruistic natives who consider migration as a poverty-alleviation device might not be the average wage for illegal immigrants. An altruistic social welfare function could for instance depend on (i) the difference between the immigrants wage and what they would have earned, had they never migrated, and/or (ii) the number of illegal immigrants (if illegal immigration is seen as the only chance of escaping poverty). In such cases, social welfare needs not depend negatively on $Z^{*}$.

${ }^{15}$ To better understand why both $\tau_{x}$ and $\tau_{a}$ are lower than $\tau_{b}$, notice that $\Omega_{x}$ and $\Omega_{a}$ have a similar structure: both are equal to $\Omega_{b}$ minus a positive function of $Z^{*}$.
} 
Welfare

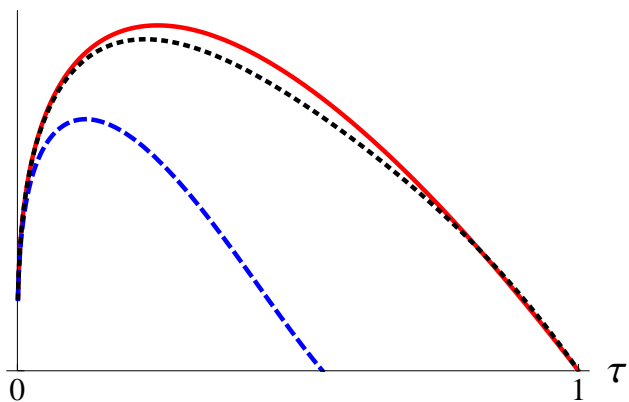

Figure 2: Social welfare functions and $\tau$ : $\Omega_{b}$ (red-solid line), $\Omega_{x}$ (bluedashed line), $\Omega_{a}$ (black-dotted line).

An interesting result of our model is that the number of illegal immigrants chosen by a welfare-maximising Government always varies in the same direction as taxes, independently of the specific welfare function. Indeed, results from Propositions 4, 5and 6, coupled with those from Lemma 2 ensure that whenever $\hat{\tau}$ is positive, $\hat{\tau} \geq \max \left\{\tau_{a}, \tau_{b}, \tau_{x}, \tau^{*}\right\}$. This implies that we are always under the case (v.2) of Proposition 2 . Therefore, our model provides an additional rationale explaining why right-wing parties, i.e. parties whose electoral platform leans towards low taxes and low public expenditures are also typically conservative on immigration matters.

Beyond taxation, in this model the Government has in principle three other policy instruments that can influence the number of illegal immigrants in the country: the probability of detection at the border $\eta$, the probability of detection of informal activities $\lambda$, and the quota of legal migrants. The following proposition summarizes the effects of changes in these variables on social welfare.

Proposition 7 Given $\rho^{*}$ and $Z^{*}$ as specified in Proposition 1 , given $\Omega, \Omega_{b}, \Omega_{x}$ and $\Omega_{a}$ as specified in Equations (24), (25), (26) and (27), then
(i) $\frac{\partial \Omega_{b}}{\partial \eta}=0, \frac{\partial \Omega_{b}}{\partial \lambda}=0, \frac{\partial \Omega_{b}}{\partial q}=0$;
(ii) $\frac{\partial \Omega_{x}}{\partial \eta}>0, \frac{\partial \Omega_{x}}{\partial \lambda}>0, \frac{\partial \Omega_{x}}{\partial q}=0$;
(iii) $\frac{\partial \Omega_{a}}{\partial \eta}>0, \frac{\partial \Omega_{a}}{\partial \lambda}>0, \frac{\partial \Omega_{a}}{\partial q}=0$; 
(iv) $\frac{\partial \Omega}{\partial \eta}>0, \frac{\partial \Omega}{\partial \lambda}>0, \frac{\partial \Omega}{\partial q}=0 ;$

Proof

See Appendix.

These results are in a sense hardly surprising, given the structure of the model. First, under the assumption that both $\eta$ and $\lambda$ are exogenous and independent of fiscal recipes, border and fiscal controls have no costs for society. Second, we have just shown here above that introducing xenophobic and/or altruistic attitudes towards illegal aliens will in any case result in less illegal immigrants. Consequently, stronger border enforcement and more detection of informal activity will always be welfare enhancing, if the size of illegal immigration enters the objective of the Government. If instead the relevant social welfare function is $\Omega_{b}$, with the Government being concerned with the utility of legal workers only, then neither $\eta$ nor $\lambda$ will affect social welfare at all, as clear from Equation (25). Finally, notice that the legal migration quota $q$ has no effect on social welfare, since in equilibrium the production function in the formal sector has constant return to labour, implying that the marginal productivity of labour is independent of the quantity of labour employed.

\section{Conclusions}

In this paper, we have built a general equilibrium model to study the interplay between illegal migration and the shadow economy in the destination country, a topic overlooked by the literature so far. In our framework, the number of illegal immigrants and the size of the informal sector are endogenously determined and may turn out to be strategic complements: the bigger the dimension of the shadow economy in the host country, the stronger the incentive for potential migrants to migrate illegally, and vice versa. Consequently, traditional determinants of informality (such as taxation and fiscal controls) can also explain illegal immigration.

This holds interesting policy implications. In particular, we have shown that, in order to contrast illegal immigration, indirect measures such as tax reduction and detection of informal activities can be used as substitutes for border enforcement. Furthermore, we have found that a welfaremaximising Government (i) will never choose to drive illegal immigration to zero, and (ii) will set the tax rate to a lower value if, rather than just focusing on the welfare of legal workers, integrates illegal immigration in its objective function. 


\section{References}

Amaral, P. S. and E. Quintin, "A Competitive Model of the Informal Sector," Journal of Monetary Economics, 2006, 53, 1541-1553.

Barro, R. J., "Government Spending in a Simple Model of Endogenous Growth," Journal of Political Economy, 1990, 98, S103-S125.

Borjas, G. J., “The Economics of Immigration," Journal of Economic Literature, 1994, XXXII, 1667-1717.

- , J. Grogger, and G. H. Hanson, "Substitution Between Immigrants, Natives, and Skill Groups," Working Paper 17461, National Bureau of Economic Research 2011.

Dabla-Norris, E., M. Gradstein, and G. Inchauste, "What Causes Firms to Hide Output? The Determinants of Informality," Journal of Development Economics, 2008, 85, 1-27.

Dell'Aringa, C. and F. Neri, "Illegal Immigrants and the Informal Economy in Italy," Labour, 1987, 1 (2), 107-126.

Djajic, S., "Illegal Immigration and Resource Allocation," International Economic Review, 1997, 38 (1), 97-117.

- and A. Vinogradova, "Undocumented Migrants in Debt," Labour Economics, 2013, 21, 15-24.

_ and _ , "Immigration Policies and the Choice between Documented and Undocumented Migration," 2015. mimeo.

Hazari, B. R. and P. M. Sgro, "The Simple Analytics of Optimal Growth with Illegal Migrants," Journal of Economic Dynamics and Control, 2003, $28,141-151$.

Ihrig, J. and K. S. Moe, "Lurking in the Shadows: the Informal Sector and Government Policy," Journal of Development Economics, 2004, 73, 541-557.

Peri, G., "Rethinking the Area Approach: Immigrants and the Labor Market in California," Journal of International Economics, 2011, 84, 1-14.

Schneider, F. and D. H. Enste, "Shadow Economies: Size, Causes, and Consequences," Journal of Economic Literature, 2000, XXXVIII, 77-114. 
Tanzi, V., "The Underground Economy in the United States: Annual Estimates, 1930-80," Staff Papers - International Monetary Fund, 1983, 30 (2), 283-305.

_ , "Uses and Abuses of Estimates of the Underground Economy," The Economic Journal, 1999, 109 (456), pp. F338-F347. 


\section{Appendix A}

\section{Proof of Proposition 1}

The model equilibria is the set of solutions $\left(\rho^{*}, Z\left(\rho^{*}\right)\right)=\left(\rho^{*}, Z^{*}\right)$ to equations (16) and (17). The first equilibrium is $(0,0)$ since $Z_{1}(0)=Z_{2}(0)$. We prove next that $Z_{1}(\rho)$ and $Z_{2}(\rho)$ coincide for a value of $\rho$ between 0 and 1 . The limits of equations (16) and (17) when $\rho$ tends to 0 and $\infty$ :

$$
\begin{gathered}
\left\{\begin{array}{l}
\lim _{\rho \rightarrow 0} Z_{1}(\rho)=0, \\
\lim _{\rho \rightarrow \infty} Z_{1}(\rho)=\infty
\end{array}\right. \\
\left\{\begin{array}{l}
\lim _{\rho \rightarrow 0} Z_{2}(\rho)=0, \\
\lim _{\rho \rightarrow \infty} Z_{2}(\rho)=0 .
\end{array}\right.
\end{gathered}
$$

On the one hand, since $Z_{2}$ is a continuous function of $\rho$, taking the value 0 for $\rho=0$ or $\rho=1$, we know that $Z_{2}$ increases first and that it has a maximum for an interior value of $\rho$. After the maximum, $Z_{2}$ decreases to reach 0 for $\rho=1$. On the other hand, $Z_{1}$ is strictly increasing in $\rho$. To prove that $Z_{1}$ and $Z_{2}$ cross at an interior value of $\rho$ we show that

$$
\lim _{\rho \rightarrow 0} Z_{1}^{\prime}(\rho)<\lim _{\rho \rightarrow 0} Z_{2}^{\prime}(\rho)
$$

Indeed,

$$
Z_{1}^{\prime}(0)=N(1+q)\left(\frac{(1-\alpha)(1-\tau) A_{F} \delta^{-\alpha}}{A_{I}(1-\lambda)[(1-\alpha) \beta+\alpha \mu]}\right)^{\frac{1}{(1-\alpha)(1-\beta)}} \frac{\alpha}{(1-\alpha)(1-\beta)}
$$

and

$$
\begin{aligned}
\lim _{\rho \rightarrow 0} Z_{2}^{\prime}(\rho) & = \\
& =\lim _{\rho \rightarrow 0} N(1+q)\left(\frac{(1-\lambda)[1-((1-\alpha) \beta+\alpha \mu)] A_{I}\left(\tau A_{F}\right)^{\frac{\alpha}{1-\alpha}} \delta^{\alpha}}{\omega+\frac{c+\eta x}{1-\eta}}\right)^{\frac{1}{\alpha+(1-\alpha) \beta}} \times \\
& \times\left(\frac{(1-\alpha) \beta}{\alpha+(1-\alpha) \beta} \rho^{\frac{(1-\alpha) \beta}{\alpha+(1-\alpha) \beta}-1}-\frac{\alpha}{\alpha+(1-\alpha) \beta}(1-\rho)^{\frac{\alpha}{\alpha+(1-\alpha) \beta}-1}\right)=\infty
\end{aligned}
$$

This finishes the proof. 


\section{Proof of Proposition 3}

The proposition demonstration is based on $Z_{2}(\rho)$ rather than on $Z^{*}$ because the expression of $Z_{2}$ is easier to handle. Proving the proposition statements for $Z_{2}(\rho), \forall \rho$, means that in particular, the statements hold for $\rho^{*}$, a particular value of $\rho$.

The partial derivatives of $Z_{2}$ with respect to $\lambda$ and $\eta$ :

$$
\begin{aligned}
\frac{\partial Z_{2}}{\partial \lambda} & =\frac{1}{(1-\lambda)(\alpha(1-\beta)+\beta)}, \\
\frac{\partial Z_{2}}{\partial \eta} & =\frac{c+x}{(1-\eta)(\alpha(1-\beta)+\beta)(c+x \eta+w(1-\eta))} .
\end{aligned}
$$

Whether $\lambda$ or $\eta$ have a larger effect on $Z_{2}$ depends on whether the changes take place on the semi-plane $\lambda>\eta$ or on the semi-plane $\lambda<\eta$ :

i) If $\lambda>\eta$, then $\frac{\partial Z_{2}}{\partial \lambda}>\frac{\partial Z_{2}}{\partial \eta}$ if

$$
1>\frac{c+x}{c+\eta x+w(1-\eta)} .
$$

Note that since $x<w$, the inequality above is trivially satisfied. Then, $\frac{\partial Z_{2}(\rho)}{\partial \lambda}>\frac{\partial Z_{2}(\rho)}{\partial \eta}$ for all $\rho$, so that $\frac{\partial Z^{*}}{\partial \lambda}>\frac{\partial Z^{*}}{\partial \eta}$.

ii) If $\lambda<\eta$, then $\frac{\partial Z_{2}}{\partial \lambda}>\frac{\partial Z_{2}}{\partial \eta}$ if

$$
\frac{1}{1-\lambda}>\frac{1}{1-\eta} \frac{c+x}{c+\eta x+w(1-\eta)}
$$

or

$$
c<\frac{w(1-\eta)^{2}+x \eta(1-\eta)-x(1-\lambda)}{\eta-\lambda}=\bar{c} .
$$

We conclude that when $\lambda<\eta, \frac{\partial Z^{*}}{\partial \lambda}>\frac{\partial Z^{*}}{\partial \eta}$ if and only if $c<\bar{c}$.

\section{Proof of Lemma 2}

The proof just follows from the direct comparison of $\hat{\tau}$ and $\tau_{b}$, for any $\hat{\tau}>0$. Notice that $\hat{\tau}>0$ if either $\alpha(1-\alpha)>\beta$, or if $\alpha(1-\alpha)<\beta$, whenever $\kappa<\frac{\alpha}{\beta(1-\alpha)-\alpha}$. 


\section{Proof of Propositions 4 and 5}

In Propositions 4 and 5 , the welfare functions $\Omega_{x}$ and $\Omega_{a}$ are defined as the difference of two other functions that we call the original functions:

$$
\left\{\begin{array}{l}
\Omega_{x}=\Omega_{b}-\theta \frac{Z^{*}}{P}, \\
\Omega_{a}=\Omega_{b}-\frac{\psi Z^{*}}{P+\psi Z^{*}}\left(w_{F}^{*}-w_{Z}^{*}\right) .
\end{array}\right.
$$

These original functions are $C^{2}$ and concave. To prove Propositions 4 and 5 we show first that the difference of two concave functions has a unique maximum. Second, we prove that the afore mentioned maximum lies below the lowest of the maxima of the original functions.

Proposition 8 Given functions $f$ and $g$, two continuously differentiable and strictly concave functions defined on $[0,1]$ such that

1) $f(0)=0, f(1)=0$,

2) $g(0)=0, g(1)=0$,

3) there exists $\tilde{\tau} \in[0,1]$ such that $f(\tau)>g(\tau)$ for all $\tau \in[0, \tilde{\tau}]$.

Let us define $W$ as their difference, $W=f-g$. Since $W$ is the difference of two concave functions, it has a unique global maximum. Moreover, if $\tau_{1}$ and $\tau_{2}$ denote the maxima of $f$ and $g$ respectively with $\tau_{1}<\tau_{2}$, then the maximum of $W, \tau^{*}$, satisfies $\tau^{*} \leq \tau_{1}$.

Proof: We shall prove first that $W$ has a unique interior maximum. Given that $f(\tau)>g(\tau)$ for all $\tau \in[0, \tilde{\tau}]$ and $f(0)=g(0)=0$, it is true that

$$
\lim _{\tau \rightarrow 0} f^{\prime}(\tau)>\lim _{\tau \rightarrow 0} g^{\prime}(\tau),
$$

so that $\lim _{\tau \rightarrow 0} W^{\prime}(\tau)>0$. Since $W\left(\tau_{1}\right)<0$ and $W$ is continuous, there exists $\tau^{*} \in\left[0, \tau_{1}\right]$ such that $W^{\prime}\left(\tau^{*}\right)=0$. Note that since $f$ and $g$ are strictly concave, $W^{\prime}$ changes sign only once in $\left[0, \tau_{1}\right]$ so that $W$ has a unique maximum, $\tau^{*}$.

Consequently, $W$ has a unique interior maximum $\tau^{*}$, with $\tau^{*}<\tau_{1}$.

Next, we prove that the couples $\left(\Omega_{b}, \theta \frac{Z^{*}}{P}\right)$ and $\left(\Omega_{b}, \frac{\psi Z^{*}}{P+\psi Z^{*}}\left(w_{F}^{*}-w_{Z}^{*}\right)\right)$ satisfy the Proposition hypothesis.

Regarding $\Omega_{b}(\tau)=A_{F}^{\frac{1}{1-\alpha}}(1-\alpha)(1-\tau) \tau^{\frac{\alpha}{1-\alpha}}, \Omega_{b}(0)=\Omega_{b}(1)=0$. Then, on the one hand, $g(\tau)$ defined as

$$
g(\tau)=\theta \frac{Z^{*}}{P}
$$


is such that $g(0)=g(1)=0$. On the other hand, defining this time

$$
g(\tau)=\frac{\psi Z^{*}}{P+\psi Z^{*}}\left(w_{F}^{*}-w_{Z}^{*}\right)
$$

it is also true that $g(0)=g(1)=0$. Hence, Proposition 8 holds for both $\Omega_{x}$ and $\Omega_{a}$, which reach a maximum which lies below $\tau_{b}$.

\section{Proof of Proposition 6}

The welfare function $\Omega$ can be written as

$$
\Omega=w_{F}^{*}-\theta \frac{Z^{*}}{P+\psi Z^{*}}-\frac{\psi Z^{*}}{P+\psi Z^{*}}\left(w_{F}^{*}-w_{Z}^{*}\right)=\Omega_{1}-\Omega_{2}-\Omega_{3} .
$$

Let us study $\Omega_{1}, \Omega_{2}$ and $\Omega_{3}$ to check whether previous results apply:

i) $\Omega_{1}$ is concave in $\tau$, with $\Omega_{1}(0)=\Omega_{1}(1)=0$.

ii) $\Omega_{2}$ is concave in $\tau$ since

$$
\frac{\partial \Omega_{2}}{\partial \tau}=\theta \frac{P}{\left(P+\psi Z^{*}\right)^{2}} \frac{\partial Z^{*}}{\partial \tau}
$$

and $\frac{\partial Z^{*}}{\partial \tau}$ is positive and then negative on $[0,1]$. Besides, $\Omega_{2}(0)=$ $\Omega_{2}(1)=0$.

iii) $\Omega_{3}$ is the product of two functions: $\frac{\psi Z^{*}}{P+\psi Z^{*}}$ and $w_{F}^{*}-w_{Z}^{*}$. The first function is concave as we have seen in point ii). Concerning the difference $w_{F}^{*}-w_{Z}^{*}$, we know that $w_{F}^{*}$ is concave in $\tau$ whereas it can be easily proven that $w_{Z}$ is increasing in $\tau$. Hence, $w_{F}^{*}-w_{Z}^{*}$ is concave and it becomes negative for a value of $\tau<1$ since

$$
\left\{\begin{array}{l}
\lim _{\tau \rightarrow 0}\left(w_{F}^{*}-w_{Z}^{*}\right)=0 \\
\lim _{\tau \rightarrow 1}\left(w_{F}^{*}-w_{Z}^{*}\right)=-\infty
\end{array}\right.
$$

We can apply Proposition 8 to the difference $\Omega_{1}-\Omega_{2}$ to deduce that the value of $\tau, \tau^{*}$, which maximizes it is smaller than the values of $\tau$ that maximize $\Omega_{1}$ and $\Omega_{2}$. It can be proven that $\Omega_{1}-\Omega_{2}$ is first concave and then convex.

Function $\Omega_{3}$ is concave: it increases first and then it decreases continuously, becoming negative at $\tau_{3}$ and tends to $-\infty$ for $\tau=1$. We make here another hypothesis : the wage of legal workers is higher than the wage 
of illegal workers in the informal sector $\left(w_{F}>w_{Z}\right)$. As a consequence, we reduce our analysis to the interval $\left[0, \tau_{3}\right]$. On this interval, $\Omega_{3}$ is positive, concave and is zero for $\tau=0, \tau_{3}$.

Summarizing, $\Omega$ is the difference of two functions. The first, $\Omega_{1}-\Omega_{2}$, has a unique interior maximum, $\tau^{*}$. It is first concave and positive, and then convex and negative. It takes value 0 for $\tau=0,1$. The second, $\Omega_{3}$ is concave. $\Omega_{3}$ dictates focusing our analysis on $\left[0, \tau_{3}\right]$, interval on which formal salaries are larger than informal salaries. As a result, applying proposition 8 on $\left[0, \tau_{3}\right]$, we can ensure that $\Omega$ has a unique interior maximum on $\left[0, \tau_{3}\right]$ which is lower than $\tau^{*}$.

\section{Proof of Proposition 7}

(i) The proof follows immediately from Equation (25): neither $\eta$ nor $\lambda$ nor $q$ appear in the expression for $\Omega_{b}$.

(ii) Because of point (i), in Equation (26) only $Z^{*}$ depends on $\eta$. Hence $\frac{\partial \Omega_{x}}{\partial \eta}=\frac{\partial \Omega_{x}}{\partial Z^{*}} \frac{\partial Z^{*}}{\partial \eta}$. From Proposition 2 we know that $Z^{*}$ always decreases in $\eta$. As $\Omega_{x}$ is decreasing in $Z^{*}$, it follows that $\frac{\partial \Omega_{x}}{\partial \eta}>0$. Using the same argument, one can prove that $\frac{\partial \Omega_{x}}{\partial \lambda}>0$. To prove that $\frac{\partial \Omega_{x}}{\partial q}=0$ notice that Equations (7) and (20) imply that the ratio $Z^{*} / P$ does not depend on $q$. Hence $\frac{\partial \Omega_{x}}{\partial q}=\frac{\partial \Omega_{b}}{\partial q}=0$.

(iii) Using the chain rule we can write $\frac{\partial \Omega_{a}}{\partial \eta}=\frac{\partial \Omega_{a}}{\partial Z^{*}} \frac{\partial Z^{*}}{\partial \eta}$. Considering Equation (28), the first partial derivative above reads

$$
\begin{aligned}
\frac{\partial \Omega_{a}}{\partial Z^{*}} & =\frac{\partial}{\partial Z^{*}}\left(\frac{-\psi}{\frac{P}{Z^{*}}+\psi}\left(w_{F}^{*}-w_{Z}^{*}\right)\right)= \\
& =\left(w_{F}^{*}-w_{Z}^{*}\right) \frac{\partial}{\partial Z^{*}}\left(\frac{-\psi}{\frac{P}{Z^{*}}+\psi}\right)+\frac{\psi}{\frac{P}{Z^{*}}+\psi} \frac{\partial w_{Z}^{*}}{\partial Z^{*}} .
\end{aligned}
$$

If $w_{F}^{*}-w_{Z}^{*}>0$, the first term in Equation (30) is negative, since

$$
\frac{\partial}{\partial Z^{*}}\left(\frac{-\psi}{\frac{P}{Z^{*}}+\psi}\right)=-\psi \frac{P / Z^{2}}{(P / Z+\psi)^{2}}<0 .
$$

To obtain the sign of 30 , it remains to compute $\frac{\partial w_{Z}^{*}}{\partial Z^{*}}$ :

$$
\begin{array}{r}
\frac{\partial w_{Z}^{*}}{\partial Z^{*}}=[(1-\alpha)(1-\beta)-1][(1-\beta)+\alpha(1-\mu)] . \\
\cdot(1-\lambda) A_{I} \delta^{\alpha} \tau^{\alpha(1+\alpha(1-\alpha))} A_{F}^{\alpha(1-\alpha)} L_{F}^{\alpha}(\rho P)^{\beta(1-\alpha)} Z^{(1-\alpha)(1-\beta)-2}<0 .
\end{array}
$$


Therefore, $\frac{\partial \Omega_{a}}{\partial Z^{*}}<0$. Since by Proposition 2, $\frac{\partial Z^{*}}{\partial \eta}<0$, it follows that $\frac{\partial \Omega_{a}}{\partial \eta}>0$.

A similar argument holds for $\frac{\partial \Omega_{a}}{\partial \lambda}>0$.

To prove that $\frac{\partial \Omega_{a}}{\partial q}=0$, consider Equation (28):

$$
\Omega_{a}=\Omega_{b}-\frac{\psi Z^{*}}{P+\psi Z^{*}}\left(w_{F}^{*}-w_{Z}^{*}\right) .
$$

We know that $\Omega_{b}, w_{F}^{*}$ and $\frac{\psi Z^{*}}{P+\psi Z^{*}}$ are independent of $q$. It turns out that $w_{Z}^{*}$ is also independent of $q$ :

$w_{Z}^{*}=[(1-\beta)+\alpha(1-\mu)](1-\lambda) A_{I} \delta^{\alpha} \tau^{\alpha(1+\alpha(1-\alpha))} A_{F}^{\alpha(1-\alpha)} L_{F}^{\alpha}\left(\rho^{*} P\right)^{\beta(1-\alpha)} Z^{*(1-\alpha)(1-\beta)-1}$.

Indeed, substituting Equations (8), (19) and (20) for $L_{F}, \rho^{*}$ and $Z^{*}$ respectively, we observe that $q$ disappears from the expression of $w_{Z}^{*}$.

(iv) $\Omega$ can be rewritten as

$$
\Omega=\Omega_{a}-\theta \frac{Z^{*}}{P+\psi Z^{*}}
$$

Hence

$$
\frac{\partial \Omega}{\partial \eta}=\frac{\partial \Omega_{a}}{\partial \eta}-\theta \frac{\partial}{\partial \eta}\left(\frac{Z^{*}}{P+\psi Z^{*}}\right)=\left(\frac{\partial \Omega_{a}}{\partial Z^{*}}-\frac{\theta P}{\left(P+\psi Z^{*}\right)^{2}}\right) \frac{\partial Z^{*}}{\partial \eta} \geq 0 .
$$

As for the other welfare functions, it is also true that $\frac{\partial \Omega}{\partial q}=0$. Regarding the effect of $\lambda$ on $\Omega$ :

$$
\frac{\partial \Omega}{\partial \lambda}=\frac{\partial \Omega_{a}}{\partial \lambda}-\theta \frac{\partial}{\partial \lambda}\left(\frac{Z^{*}}{P+\psi Z^{*}}\right)=\frac{\partial \Omega_{a}}{\partial \lambda}-\frac{\theta P}{\left(P+\psi Z^{*}\right)^{2}} \frac{\partial Z^{*}}{\partial \lambda} \geq 0 .
$$




\section{Appendix B}

\section{Endogenous $\lambda$ and $\eta$ : a numerical exercise}

So far, we have assumed that the probability of detection of informal activity, $\lambda$, and the probability of detection at the border, $\eta$, are not interconnected and do not depend on fiscal recipes. Although these assumptions are obviously not realistic, still they were necessary in order to solve the model analytically. In this section, we remove these assumptions and run a numerical exercise with a more general version of the model. The objective is to see whether the main conclusions from the previous sections hold, and what new insights, if any, we might get. Results show that the existence of an additional trade-off between border patrolling and detection of informal activities enriches significantly the model.

We introduce two main modifications to the benchmark model of Section 2. First, we introduce a new variable, the police ( $C$ for cops), which we assume is financed through tax revenues. Therefore, the policy maker must choose whether to use fiscal recipes to finance public production or to hire more policemen. Denoting by $\chi$ the share of tax revenue assigned to financing public expenditures, we have

$$
\begin{array}{r}
G=\chi \tau Y_{F}, \\
C=(1-\chi) \tau Y_{F} .
\end{array}
$$

Second, the policy maker faces another choice, namely how to split the Police force between border patrolling and detection of informal activities. We assume that the number of policemen, $C$, is the main determinant of both the probability of detection of informal activity, $\lambda$, and the probability of detection at the border, $\eta$. In particular, we assume that both are increasing and concave functions of the quantity of police employed. In the simulations, we have specified the following functional forms:

$$
\begin{gathered}
\eta=B \frac{\xi C}{1+\xi C^{\prime}} \\
\lambda=\frac{(1-\xi) C}{1+(1-\xi) C^{\prime}}
\end{gathered}
$$

where $B$ is a technological parameter, and $\xi$ is the share of the Police force allocated to border patrolling.

These modifications make the model not solvable analytically. We therefore resort to numerical analysis. This implies that we have to assign 
numerical values to the exogenous variables and to the parameters of the model.16

We normalize the native population to 1 .

The parameter $\alpha$ is fixed to 0.25 as in Barro (1990).

The parameter $\beta$ in the labour aggregator (9) is fixed to 0.5 , so that both legal and illegal workers have the same labour share in the production function (4).

We assume that TFP in the formal sector, $A_{F}$, is higher than TFP in the informal sector, $A_{I}$. We normalize the latter to 1 , and set $A_{F}$ to 2 .

We assume that the cost of migration, $c$, and the return penalty, $x$, each amounts to $25 \%$ of the wage in the sending country, $\omega$. The latter is kept low enough to ensure that $\omega<w_{F}$.

We assume that the share of public goods available to the firm in the informal sector is 0.8 .

The share of the marginal productivity of public expenditures in the informal sector grasped by legal workers, $\mu$, is fixed to 0.5 . This implies we assume there is no 'exploitation': legal and illegal workers get the same share of the marginal productivity of $G$.

The productivity parameter $B$ is set to 1 . That is, we are assuming that policemen are equally productive in detecting informal activities and patrolling borders.

The simulation exercise consists in studying how the equilibrium $\left(\rho^{*}, Z^{*}\right)$ is affected by variations in the choice variables of the Government, namely how many resources are assigned to police, $(1-\chi)$, and how many policemen are assigned to border patrolling, $\xi$. To help the interpretation of the results, we also report variations in the equilibrium values of $\eta$ and $\lambda$. We run the simulations for three possible fiscal scenarios: a low-tax country $(\tau=10 \%)$, a country with taxes averaging around the Continental European level $(\tau=40 \%)$, a high-tax country $(\tau=70 \%)$. Results from the simulations are shown in Figures 3,4 and 5 .

As one might have expected, $\rho^{*}$ and $Z^{*}$ are increasing in $\chi$ in all scenarios. The higher the amount of public resources allocated to the production of the public good, the bigger the dimension of the shadow economy, the higher the call effect on illegal immigrants.

\footnotetext{
${ }^{16}$ Given the illustrative (e.g. qualitative) nature of our exercise, we are not calibrating the model stricto sensu. Rather, the choice of the values of the parameters is mainly based on plausibility. Consequently, although some robustness analysis is discussed in the Appendix, the results of our exercise could not be intended as exhaustive, especially from a quantitative point view. We claim nonetheless that the main conclusions that we draw from the simulations in terms of positive analysis and policy choices are robust to a wide range of values for the parameters.
} 
Somewhat more surprisingly, $\rho^{*}$ and $Z^{*}$ are also increasing in $\xi$ in all scenarios. We have an apparent paradox here: for any given amount of public resources assigned to the police, the higher the number of policemen the Government employs to control the border, the higher the resulting number of illegal immigrants in the country. The rationale for this result is that there is a trade off between sending policemen to the border or sending them to detect informal activities. Any increase in border patrolling has the side effect of reducing detection of informal activities, which in turn increases the returns to the informal sector. Accordingly, the incentive compatibility constraint for potential illegal immigrants, Equation (15), becomes less binding: the economy will end up having a bigger shadow economy and more illegal immigrants ${ }^{17}$ This suggests that policies aimed at reducing illegal immigration should not directly target border control.

How well do these result compare with those obtained in Section 2? There, we showed that the Government has four effective instruments to reduce the number of illegal immigrants: the immigration quota, $q$, the detection of informal activities, $\lambda$, the control of the frontiers, $\eta$, and tax rate, $\tau$. The numerical results for the more general version of the model here show that once the trade off between $\eta$ and $\lambda$ is taken into account, increases in the resources allocated to border patrolling cannot be considered an effective way of reducing illegal immigration anymore.

\footnotetext{
${ }^{17}$ Notice that this result also depends on two specific assumptions. First, in this model the Inada conditions always hold. The marginal productivity of labour by illegal immigrants goes to infinity as their number approaches to zero. This implies that zero illegal immigration cannot be a solution of the model. Second, the equations in Formula (33) imply that while $\eta$ and $\lambda$ can both drop to 0 , they are instead bounded from above, and therefore strictly less than 1 . Consequently, in this model there cannot be full control by the State of either illegal immigration or of the shadow economy.
} 

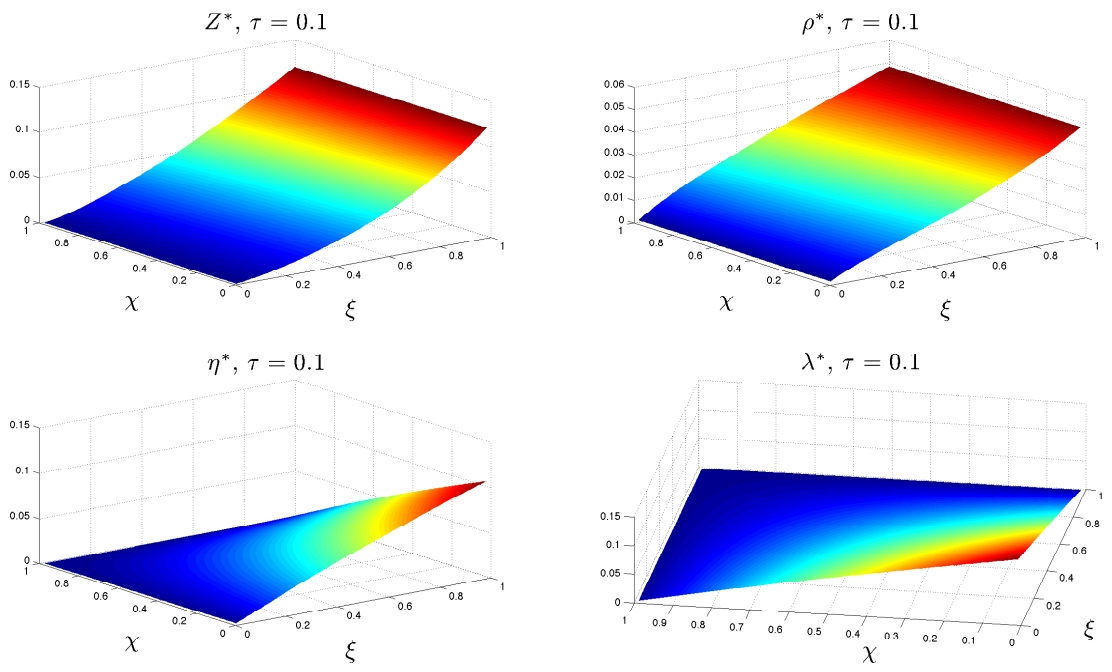

Figure 3: Simulation of the model in Section $4: \tau=0.1$.
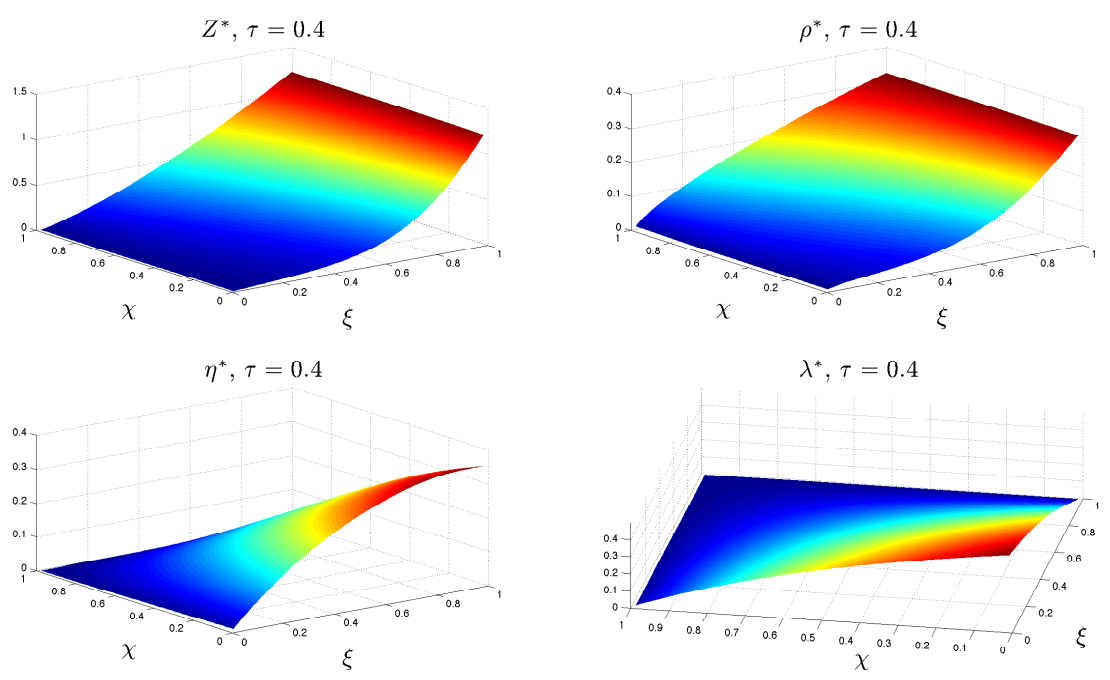

Figure 4: Simulation of the model in Section $4: \tau=0.4$. 


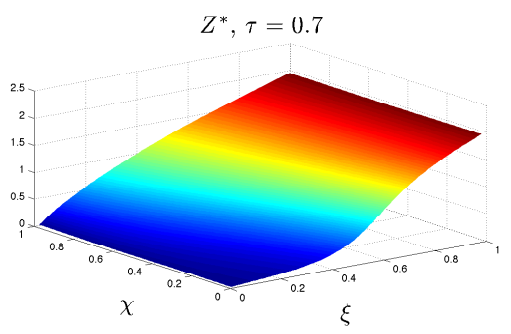

$\eta^{*}, \tau=0.7$

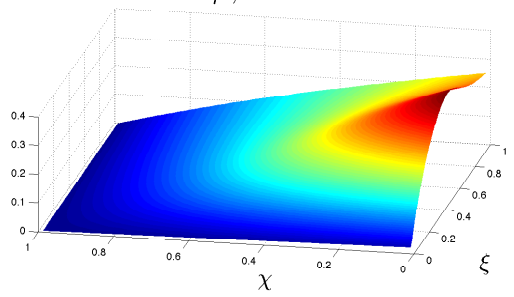

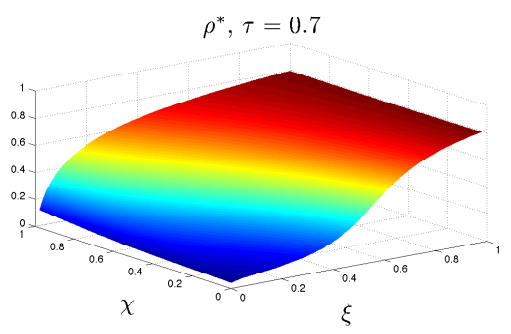

$\lambda^{*}, \tau=0.7$

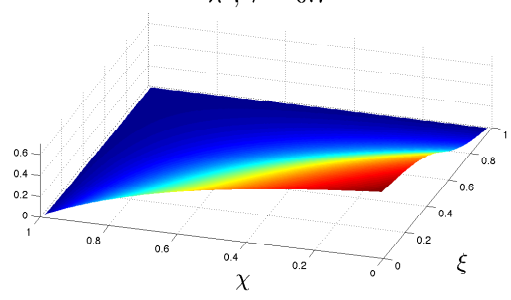

Figure 5: Simulation of the model in Section $4: \tau=0.7$. 
Institut de Recherches Économiques et Sociales

Université catholique de Louvain

Place Montesquieu, 3

1348 Louvain-la-Neuve, Belgique 